\title{
Demencia frontotemporal: revisión y nuestro punto de vista
}

\author{
Ildefonso Rodríguez-Leyva, José Eduardo Oliva-Barrios, Santiago Cueli-Barcena, \\ Juan Carrizales-Rodríguez, Erika Chi-Ahumada y María E. Jiménez-Capdeville \\ Servicios de Neurologia y Bioquímica, Facultad de Medicina, Universidad Autónoma de San Luis Potosí, SLP, México
}

\begin{abstract}
Resumen
La demencia frontotemporal (DFT) constituye un grupo de enfermedades neurodegenerativas con características clínicas, fisiopatológicas y anatomopatológicas particulares. Son un verdadero reto para los médicos, y no se produce el suficiente avance en el conocimiento para evitarlas, detener su progresión y/o proporcionar un tratamiento específico. Ahora nos limitamos a dar un diagnóstico elegante con un pronóstico sombrío. Es la demencia neurodegenerativa que comienza en personas menores de 65 años. Las principales características clínicas son manifestaciones conductuales y del lenguaje que definen y permiten clasificar este trastorno. Las proteinopatías involucradas son p-Tau, TDP-43 y FUS. Las mutaciones más relacionadas ocurren en los genes tau (MAPT), progranulina (PGRN), especialmente el C9orf72 y otros genes menos comunes. La imagen característica es una atrofia lobar frontotemporal que podemos observar en una tomografía computarizada y aún mejor en una resonancia magnética (RM) cerebral. Con algunos estudios funcionales en particular, como la RMf (resonancia magnética funcional), tomografía computarizada por emisión de fotón único (SPECT) o tomografía por emisión de positrones (PET), es posible determinar los patrones característicos de anormalidades de los diferentes subtipos de demencias frontotemporales (DFT). La acumulación de tau puede determinarse por imagen, y hay cambios especificos en las sustancias gris y blancas que se pueden identificar en los subtipos de DFT. No hay un tratamiento específico disponible, y sólo se prescriben sintomáticos para la cognición, depresión, psicosis, crisis epilépticas, alteraciones en el sueño y/o pérdida en la continencia de las emociones, tratando de mejorar la calidad de vida de los pacientes, y para ayudar a la familia y al cuidador en su manejo. Realizar un diagnóstico temprano mediante técnicas novedosas y accesibles es el inicio para encontrar formas de prevención. Una opción que propone nuestro grupo de investigación consiste en estudiar la piel de los pacientes afectados buscando la presencia de las proteinopatías cerebrales reflejadas en la piel. Esta técnica puede no sólo apoyar el diagnóstico, sino también ayudar a comprender la fisiopatología del problema e incluso evaluar una respuesta terapéutica.
\end{abstract}

Palabras clave: Demencia frontotemporal (DFT). Variante conductual de DFT (DFTvc). Afasia progresiva primaria (APP). Tau. TDP-43. C9orf72. Expresión en la piel.

\section{Frontotemporal dementia: revision and our point of view}

\section{Abstract}

Frontotemporal lobar dementia is a group of neurodegenerative diseases with clinical, physiopathological and anatomopathological particular characteristics. They are a challenge, and there is not enough advance in how to avoid them, how to

\section{Correspondencia:}

Ildefonso Rodríguez-Leyva

E-mail: ilrole@yahoo.com.mx
Fecha de recepción: 23-08-2018

Fecha de aceptación: 11-09-2018

DOI: 10.24875/RMN.M18000011
Disponible en internet: 03-01-2019 Rev Mex Neuroci. 2018;19(6):20-31 www.revmexneurociencia.com 1665-5044/@ 2018. Academia Mexicana de Neurología A.C. Publicado por Permanyer México. Este es un artículo Open Access bajo la licencia CC BY-NC-ND (http://creativecommons.org/licenses/by-nc-nd/4.0/). 
make an early diagnosis, how to stop the progression and how to give them a specific treatment. Now our limit is only to give an elegant diagnosis with a hopeless prognosis. This dementia starts in younger than 65-year-old. The principal clinical characteristics are behavioral and language manifestations that define and classified this problem clinically. The proteinopathies involved are p-Tau, TDP-43, and FUS. The genetic mutations are related to the tau gene (MAPT), the progranulin gene, especially the C9orf72 and others. The image is frontotemporal lobar atrophy that we can follow out with computed tomography scan or magnetic resonance imaging (MRI) of the brain. With some specific MRI, SPECT or positron emission tomography functional studies are possible to determinate the characteristic patterns of abnormalities of the different subtypes of discrete fourier transform (DFT). Accumulation of tau is visible by image and specific changes in grey and white matter in the different subtypes of DFT. There is not a specific treatment; we use symptomatic drugs for cognition, depression, psychosis, seizures, sleep disorders, loss in the continence of the emotions, trying to improve the quality of life of the patient and help to the family and the caregiver in the manage. To find new and more ways to make an early diagnosis is the beginning of find possibilities for prevention. Our research group is proposing the study of the skin on affected patients looking for the presence of the same proteinopathies in the skin that is present in the brain. With this technique, there can be the possibility not only to support the diagnosis but also understand the pathophysiology, and inclusive evaluate the therapeutic answer.

Key words: Frontotemporal dementia (DFT). DFT behavioral variant (DFTvc). Primary progressive aphasia (APP). Tau TDP-43. C9orf72. Expression in the skin.

\section{Introducción}

Las DFT son un grupo de enfermedades neurodegenerativas caracterizadas por cambios importantes en la conducta o pérdida progresiva del lenguaje asociados con alteraciones patológicas localizadas en los lóbulos temporales y frontales del cerebro. El término degeneración lobar frontotemporal (DLFT) se utiliza para aquellos pacientes que clínicamente presentan la enfermedad y tienen evidencia genética o histopatológica (biopsia 0 post mortem) de DFT. La DFT es conocida también como el «desorden de tres», por su asociación a tres síndromes clínicos, tres variantes histopatológicas y tres alteraciones genéticas. Es la tercera forma más común de demencia después de la enfermedad de Alzheimer (EA) y la demencia con cuerpos de Lewy (DCL)1.

\section{Epidemiologia}

La DFT se manifiesta a los 45-65 años de edad. Según la Organización Mundial de la Salud, se estima que hay 47,5 millones de personas que padecen demencia y otros 7,7 millones de casos nuevos cada año. En México no existen estadísticas de su prevalencia e incidencia. La EA es la forma más común de demencia, que contribuye al $60-70 \%$ de los casos, la DFT es la segunda causa más común de demencia, aunque en sujetos menores de 65 años $^{2}$ es la primera causa de demencia: el $10 \%$ ocurre en sujetos menores de 45 años y el $30 \%$ en mayores de 65 años. Si bien se ha considerado una enfermedad infrecuente, ahora se reconoce y diagnostica más; sin embargo, el diagnóstico no es sencillo y el abordaje clínico en ocasiones no es suficiente ${ }^{3}$.

\section{Caso clínico}

MAV mujer de 71 años de edad, diestra, licenciada en contabilidad, aunque en ese momento se dedicaba a las labores del hogar. Acude a consulta el día 09.04.18 por un cuadro, iniciado aparentemente en diciembre del año anterior, en el que la familia nota que tenía un llanto más fácil y se mostraba más callada de lo que habitualmente solía ser. Aunque come y duerme bien, el cambio conductual se asocia a pérdida del lenguaje, pues parecería no encontrar las palabras que quería expresar, mostrando falta de fluencia verbal; además, le costaba trabajo encontrar incluso el nombre de sus nietos y cometía errores frecuentes al nominar o intentar expresar algo.

Respecto a los antecedentes importantes, destaca hipertensión, motivo por el cual toma Cozaar (Iosartán), le quitaron un fibroadenoma de la mama izquierda y le practicaron abdominoplastia estética y una hernioplastia. Niega tabaquismo y etilismo. Tuvo cinco gestaciones, de las que tiene cinco hijos aparentemente sanos.

Anteriormente había sido estudiada médicamente en las ciudades de Monterrey y Guadalajara, en donde se le hizo imagen por resonancia magnética (IRM) de cráneo y columna cervical, y recibió tratamiento antiparkinsoniano.

La IRM de cráneo de tres meses antes muestra atrofia lobar frontotemporal bilateral y asimétrica, con importante afección de las áreas frontal y temporal (principalmente notable a nivel insular) del lado izquierdo (Fig. 1).

Sus constantes vitales son: TA de 150/90, FC de 68 latidos por minuto, frecuencia respiratoria (FR) de 21 por minuto, $\mathrm{T}$ de $36.2^{\circ} \mathrm{C}$, peso de $78 \mathrm{~kg}$ y talla de $165 \mathrm{~cm}$. La 


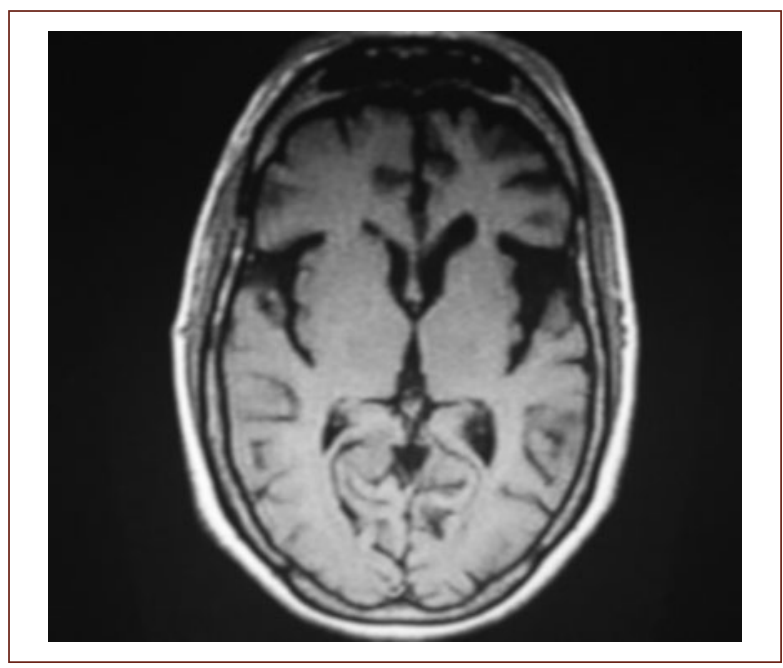

Figura 1. Imagen de resonancia magnética T1 que muestra la atrofia frontotemporal característica con asimetría específica acorde con una APP en la paciente del caso clínico presentado como ejemplo de la enfermedad.

exploración general prácticamente no aporta datos relevantes, solamente un moderado sobrepeso (IMC de 28.65)

La exploración neurológica muestra un MMSE de 21 puntos, con problemas especialmente en la fluencia y la nominación, agramatismo y parafasias fonológicas, acalculia y apraxia idiomotora, aunque parece comprender; lee y escribe. La exploración de los nervios craneales fue normal, solamente llamó la atención el hecho que parpadeaba poco. En el sistema motor sólo se observa discreta bradicinesia; el tono, la masa, la fuerza y los reflejos están conservados. Aparentemente no hay alteraciones sensitivas y no se encuentran signos cerebelosos ni meníngeos.

Por el cuadro clínico y la imagen, concluimos que la paciente estaba cursando con una DFT en su variante de afasia progresiva primaria (APP) tipo no fluente, lo que fue corroborado por la evaluación neuropsicológica.

Sus estudios de laboratorio se encontraron dentro de los límites normales. No se realizaron electroencefalograma, pruebas genéticas ni biopsia de piel.

El diagnóstico sugiere que, desafortunadamente, la paciente estaba cursando con un proceso neurodegenerativo, que su manejo sería principalmente sintomático y su pronóstico desfavorable a mediano plazo.

La paciente es manejada con fluoxetina, memantina y rivastigmina, que aparentemente tolera y que por lo menos estabilizan su condición, aunque podría ser controversial el manejo con anticolinesterásicos. Su último MMSE, realizado el 02.08.18, subió dos puntos en relación con el anterior.

\section{Síndromes clínicos}

El espectro clínico incluye: a) DFT en su variante conductual (DFTvc), b) APP variante semántica (APPvs) y c) APP no fluente. Cada una tiene unas características clínicas que la distinguen de las otras dos; sin embargo, conforme la enfermedad progresa, pueden traslaparse entre ellas.

En general, sus principales características clínicas consisten en cambios progresivos en el comportamiento y la personalidad, disfunción ejecutiva y alteración progresiva en el lenguaje ${ }^{4}$.

\section{Demencia frontotemporal variante conductual}

Es la variante más común; aproximadamente el $50 \%$ de los casos.

\section{Síntomas}

Se observan cambios sutiles en la personalidad y la conducta que progresan con el tiempo. La apatía es el síntoma inicial más común, aunque también se producen pérdida en la empatía y simpatía hacia los demás, conductas obsesivas y estereotipadas, y cambios en la dieta como glotonería y preferencia por los alimentos dulces. Los pacientes afectados muestran dificultad para la resolución de problemas y para tener flexibilidad mental, y el razonamiento abstracto también se encuentra alterado. Algunos pacientes pueden manifestar síntomas psicóticos como ilusiones y alucinaciones, con cierta frecuencia se desinhiben y se muestran hipersexuales, y hacen comentarios inapropiados en momentos inadecuados.

\section{Examen neurológico}

En estos pacientes se observan síntomas de afección del lóbulo frontal anterior. Su aspecto personal es de mal aseo e higiene, se comportan de manera inadecuada socialmente y se muestran apáticos, y en la exploración neurológica pueden encontrarse signos de liberación frontal (signo de la glabela, búsqueda, chupeteo, prensión, palmomentoniano, etc.). Se observa un déficit importante en las funciones ejecutivas, no así en la memoria ni en la capacidad visuoespacial. Se suele encontrar a un sujeto perseverativo, confabulatorio e impulsivo, así como con dificultad para reconocer las expresiones faciales. Si mantiene la fluidez fonológica y semántica, puede incluir palabras obscenas de manera repetitiva ${ }^{5}$. 


\section{Afasia progresiva primaria variante no fluente agramatical}

Es un desorden frecuentemente asociado a una atrofia asimétrica, generalmente mayor del hemisferio izquierdo en comparación con el derecho.

\section{Síntomas}

Los pacientes con esta variante tienen conservan la memoria, la función visuoespacial y su comportamiento, pero tienen pérdida progresiva en la capacidad para la expresión verbal. El habla no es fluida y se lentifica, se producen agramatismos, parafasias fonológicas, apraxia en el discurso, anomia y les cuesta encontrar las palabras adecuadas. La comprensión del lenguaje se mantiene por más tiempo, pero finalmente se pierde. Otros déficits asociados son la apraxia idiomotora y la acalculia.

\section{Examen neurológico}

El paciente presenta pérdida de la fluidez verbal, dificultad para las encontrar palabras, tartamudea y tiene errores gramaticales, con una relativa preservación de la comprensión.

Puede haber un sutil déficit motor del lado derecho, especialmente si hay extensión del involucramiento del lóbulo frontal izquierdo.

Pueden encontrarse signos atávicos y en ocasiones de parkinsonismo (temblor, rigidez, bradicinesia e inestabilidad postural). Los pacientes con diagnóstico de parálisis supranuclear progresiva (PSP) y degeneración corticobasal (DCB) pueden cumplir todas las características de este problema asociado a su parkinsonismo de base. Para el diagnóstico se requiere al menos una de las dos características principales (agramatismo en la producción del lenguaje o esfuerzo e interrupción del mismo, no congruente con la apraxia del habla) y dos de las siguientes características: a) comprensión limitada de oraciones complejas, b) comprensión limitada de una palabra aislada y c) no reconocimiento de objetos aislados ${ }^{6}$.

\section{Afasia progresiva primaria variante semántica}

\section{Síntomas}

Esta variable también se asocia a síntomas relacionados con el lenguaje, particularmente la presencia de anomia. Los pacientes pueden tener un discurso fluente, estructurado desde el punto de vista sintáctico y fonológico, pero tienen problemas para darle significado a las palabras. Conforme avanza la enfermedad van perdiendo la capacidad de darle la traducción conceptual a los objetos. Conservan la memoria, la orientación visuoespacial y la memoria autobiográfica, pero no expresan parafasias fonémicas, como ocurre en la variante no fluente.

\section{Examen neurológico}

El paciente muestra dificultad para dar y recibir información al conversar, comportamiento anormal debido al involucramiento del lóbulo temporal anterior derecho y sus conexiones con la corteza orbitofrontal. Si la región posterior temporal y las áreas visuales de asociación están afectadas, puede desarrollar agnosia visual y aprosopagnosia (incapacidad para reconocer los rostros).

El resto del examen puede ser normal, incluyendo los reflejos frontales, que se aprecian más tardíamente.

El diagnóstico de esta variante se hace cuando los pacientes muestran nomenclatura de confrontación alterada (es decir, dificultad para nombrar o reconocer objetos o dibujos), dificultad en la comprensión de una sola palabra y si tienen al menos tres de los siguientes problemas: a) conocimiento del objeto deteriorado, b) dislexia superficial (es decir, incapacidad para reconocer palabras al leerlas como un todo) o disgrafía (al escribirlas), c) repetición alterada y d) deterioro en la producción de habla espontánea?.

\section{Afasia progresiva logopénica}

Es una afección del lenguaje con características distintivas, pero no tan común como las otras dos variantes de APP. En esta forma de presentación los pacientes no pierden el significado de las palabras, como en la variante semántica, ni se ve afectada la pronunciación, como en la variante no fluente. Los pacientes con afasia logopénica exhiben deterioro del lenguaje, pero con dificultades más específicas en nominar y en la sintaxis de las oraciones, cuya característica más prominente es la dificultad para encontrar las palabras. Estas personas tienen más probabilidades de tener un lenguaje interrumpido con sonidos para llenar las frecuentes pausas, los errores fonológicos y la repetición de las oraciones mal conformadas. Su lenguaje suele ser fluido, pero puede confundirse con la variable no fluente debido a que el paciente habla lento y hace 
pausas al no encontrar las palabras que va a utilizar para expresarse. Esta variante puede llegar a ser un síntoma de inicio focal de la EA, ya que en estudios de patología post mortem se ha encontrado que un $46 \%$ de ellas corresponden a EA. También puede estar mezclada clínica y patológicamente con una DCL en el $8 \%$ de los casos, ser una DLFT en el $23 \%$ u otras enfermedades con un fenotipo similar en el $23 \%$.

El diagnóstico de la variante logopénica requiere la presencia de las siguientes características: incapacidad para la recuperación de una sola palabra en el lenguaje espontáneo, e imposibilidad para la repetición de oraciones y frases. Además de tres de las cuatro siguientes características para un diagnóstico más certero: a) errores fonológicos en el habla y al nombrar con palabras espontáneas, b) comprensión alterada de palabras sueltas y objetos, $c$ ) tener un discurso motor perdido y d) presentar agramatismo franco ${ }^{8}$.

\section{Otras formas de presentación}

La DFT puede estar asociada a otros síndromes clínicos que, por su extensión y características, no tocamos en esta revisión, como la asociación con la PSP o con la DCB, ambas taupatías caracterizadas por parkinsonismo. La primera se asocia a limitación de la mirada vertical y caídas tempranas, y la segunda a un síndrome de mano ajena (alienígena) y, si es izquierda, a APP.

\section{Histología}

La acumulación de las proteínas p-Tau y TDP-43 (transactive response DNA binding protein $43 \mathrm{kDa}$ ) caracterizan el $90 \%$ de los hallazgos patológicos confirmatorios de DLFT; el 10\% restante corresponde a acumulación de la proteína FUS (fused in sarcoma RNA binding protein $)^{9}$.

\section{Factores genéticos implicados}

En la DFT se puede encontrar un patrón autosómico dominante, en el que existen familiares de primer grado afectados ( $40 \%$ de los casos).

Existen al menos ocho genes implicados, de los cuales tres son las principales mutaciones (50\%): el gen de la proteína tau asociada a microtúbulos (MAPT) en el cromosoma 17, el gen de la proglanulina (PGRN), también relacionado al cromosoma 17, y el gen C9orf72, asociado al cromosoma $9^{10}$.

\section{Correlación clínico-genética}

De acuerdo a la presentación clínica (fenotipo) se puede inferir el genotipo. La variante conductual se puede asociar a mutaciones en cada uno de los genes (no es discriminatoria). Sin embargo, en pacientes con la mutación en MAPT la desinhibición conductual es la característica, mientras que con la mutación en granulina (GRN) y C9orf72 la apatía es la manifestación típica.

Las mutaciones en GRN están asociadas con la variante no fluente en un tercio de los casos, dejando los otros dos tercios para la variable conductual. La variable no fluente no se relaciona con MAPT y es raro que se relacione con C9orf72. Las características psicóticas son más frecuentes en pacientes con expansiones en C9orf72 ${ }^{11}$.

\section{Correlación genético-patológica}

\section{Gen tau (MAPT)}

El gen tau (MAPT) se ubica en 17q21-22. Se ha encontrado que más de 30 diferentes mutaciones en este gen se relacionan con la DFT. Todas las mutaciones se encuentran localizadas entre los exones 9 y 13, y dentro de estas mutaciones se relacionan a un aumento desproporcionado de las isoformas $3 R$ y $4 R$, que ocasionan cambios en la afinidad de la unión de la tubulina y la actividad estabilizante de los microtúbulos.

Cualquier exceso de proteína pTau (tau fosforilada) es empaquetado como residuo no digerible, que conforma cuerpos de inclusión (cuerpos de Pick conformados por marañas neurofibrilares, diferenciables de las de la EA). Los portadores de mutaciones en MAPT se caracterizan por tener depósitos de proteínas tau insolubles y agregadas dentro de las neuronas, células gliales de la corteza cerebral y otras regiones.

El fenotipo clínico de mutaciones de este gen es heterogéneo, pero los cambios conductuales, la discapacidad semántica, la pérdida de la memoria episódica y el parkinsonismo han sido propuestos como las claves sugerentes de las manifestaciones clínicas ${ }^{12}$.

\section{Gen de la progranulina (PGRN)}

La progranulina y la granulina tienen un papel de remodelación en los tejidos. La progranulina es expresada en las neuronas y en la microglía. El gen está ubicado en 17q21, y esta mutación aparece en el $5-20 \%$ de los pacientes con historia familiar 
positiva y el $1-5 \%$ se relaciona con los de aparición esporádica. Mutaciones en este gen generan codones de paro prematuros, que causan pérdida de hasta el $50 \%$ de la proteína codificada, lo que lleva a una enfermedad por haploinsuficiencia caracterizada por neuroinflamación con microgliosis y astrogliosis en la corteza, los hipocampos, los tálamos y el tallo cerebral.

Los portadores de esta mutación parecen presentar el fenotipo clínico más variado. La disfunción en el lenguaje es común mientras la enfermedad avanza. Los síntomas extrapiramidales se pueden presentar en un $40-60 \%$ de los casos $^{13}$.

\section{Gen C9orf72 9 (marco de lectura abierta 72 en el cromosoma 9)}

Codifica para una proteína citoplasmática neuronal localizada en terminales presinápticas. Mutaciones en este gen parecen ser la anomalía genética más común para la DFTvc (11\%) y para la demencia asociada a la esclerosis lateral amiotrófica (23\%).

Los portadores de la expansión de este gen presentan con mayor frecuencia brotes de psicosis, con alucinaciones e ilusiones ${ }^{14}$.

\section{Otros genes implicados}

Se han asociado a la DLFT otras mutaciones, como la del gen VCP (que codifica a la proteína que contiene valosina), asociado a la tríada clínica de demencia lobar frontotemporal, miopatía por cuerpos de inclusión y enfermedad de Paget $^{15}$.

\section{Pruebas genéticas}

Se realizan las pruebas genéticas para buscar mutaciones de C9orf72, MAPT y PGRN.

Otros biomarcadores son la proteína $\beta$ amiloide 42 disminuida y el aumento de la proteína $p$-Tau (Tau fosforilada) en el LCR, que tiene una sensibilidad y especificidad aproximada del $80 \%$ para la EA, mientras que en la demencia lobar frontotemporal los niveles de tau suelen ser normales o incluso bajos, y los de $\beta$ amiloide 42 son más altos que en el Alzheimer. Los niveles séricos de progranulina pueden manifestar la mutación de GRN en portadores y pacientes. Se encuentran aún en proceso de investigación marcadores en el LCR de cadenas ligeras de neurofilamentos para TDP-43 y progranulina $^{16}$.

\section{Neuroimagen}

El hallazgo característico de la DFTvc en estudios de neuroimagen es atrofia o hipometabolismo del lóbulo frontal o temporal anterior derecho.

Recientemente se han empleado nuevos métodos diagnósticos con diferentes modalidades de imagen (tractografía, PET, RM funcional, entre otras), las cuales han permitido la posibilidad de investigar las alteraciones estructurales y funcionales en la conectividad cerebral, y han ayudado en la visualización de la deposición patológica de las proteínas implicadas en esta enfermedad.

Los primeros estudios de neuroimagen que cuantifican la atrofia de IRM e hipometabolismo, y que han usado la PET con 18F-fluorodesoxiglucosa (FDG-PET), han revelado patrones característicos de anomalías en los subtipos de DFT.

Los avances recientes en nuevas modalidades de imágenes, como la imagen de tensor de difusión (DTI), la RM funcional en estado de reposo, la angiografía no contrastada (ASL) y la PET-tau, proporcionan resultados prometedores y nuevas técnicas para investigar la conectividad y los cambios moleculares en estos subgrupos clínicos, genéticos y patológicos ${ }^{17}$.

\section{Neuroimagen en la demencia frontotemporal}

Los pacientes con mutaciones MAPT presentan principalmente atrofia focal y simétrica del lóbulo temporal anterior y lóbulo orbitofrontal. Las mutaciones GRN comúnmente se presentan con una atrofia marcada asimétrica de las regiones parietal y frontal inferiores. Y las mutaciones C9orf72 muestran un patrón de atrofia simétrico más distribuido que afecta predominantemente a los lóbulos frontales en las regiones dorsolaterales y mediales, así como en la región orbitofrontal, con pérdida adicional en los lóbulos temporal anterior, parietal y occipital, así como los tálamos y el cerebelo.

En las taupatías, la enfermedad de Pick puede asociarse con atrofia asimétrica a menudo en la corteza frontal (incluidas las regiones orbitofrontal, medial y dorso lateral principalmente), con afección de los lóbulos temporales anteriores y la ínsula.

La RMf en estado de reposo puede ser usada para dilucidar la función de las redes sinápticas conectadas entre sí midiendo fluctuaciones sincronizadas dependientes del tiempo en los niveles de oxigenación de la sangre (señal BOLD), como una medida sustituta de la actividad neuronal coordinada. 
Rev Mex Neuroci. 2018;19

Tabla 1. Criterios diagnósticos para la DFTvc

Criterios diagnósticos

Consenso internacional de criterios para el diagnóstico de la DFTvc

Enfermedad neurodegenerativa: comienzo insidioso, y deterioro progresivo y gradual de la conducta o cognición

bvDFT posible:

- Síntomas persistentes o recurrentes de las siguientes manifestaciones

- Al menos tres de los siguientes síntomas:

- Conducta desinhibida al inicio de la enfermedad (al menos uno de los siguientes):

- Comportamiento social inadecuado

- Pérdida de modales o conductas de comportamiento social

- Acciones impulsivas o precipitantes

- Apatía o inercia al inicio de la enfermedad (al menos uno):

- Apatía

- Inercia

- Pérdida de empatía o simpatía (al menos uno):

- Respuesta disminuida a las necesidades o sentimientos de otras personas

- Disminución del interés por actividades sociales, interrelaciones o calidez personal

- Comportamiento perseverativo, estereotipado o compulsivo/ritualista (al menos uno):

- Movimientos simples repetidos

- Comportamiento ritualista, complejo o compulsivo

- Estereotipia del lenguaje

- Hiperoralidad y cambio de las costumbres alimenticias (al menos uno):

- Cambios en las preferencias alimentarias

- Atracones

- Exploración bucal o consumo de objetos no comestibles

- Perfil neuropsicológico alterado, con todos los siguientes:

- Déficit en las funciones ejecutivas

- Preservación relativa de la memoria episódica

- Preservación de las habilidades visuespaciales

bvDFT probable:

- Todos los siguientes deben estar presentes:

- Cumple con criterios para bvDFT posible

- Deterioro funcional significativo

- Estudios de imagen consistentes con bvDFT (al menos uno):

- Atrofia frontal o temporal en RM o TC de cráneo

- Hipoperfusión en PET o hipometabolismo en SPECT frontal o temporal anterior

bvDFT con enfermedad definitiva de degeneración lobar frontotemporal:

- Debe contar con el criterio 1, además del criterio 203 :

1. Cumple con criterio para bvDFT posible o probable

2. Evidencia histopatológica de DLFT en biopsia o post mortem

3. Presencia de mutación patogénica conocida

Criterios de exclusión

- Los criterios 1 o 2 deben ser contestados de forma negativa para cualquier diagnóstico de bvDFT

- El criterio 3 puede ser positivo para posible bvDFT y negativo para probable bvDFT

1. El patrón de déficit es mejor explicado por otra afección médica o del sistema nervioso no degenerativa

2. Los cambios conductuales se explican mejor por un diagnóstico psiquiátrico

3. Los biomarcadores sugieren fuertemente EA u otro proceso neurodegenerativo

bvDFT: Variante conductual de la DFT. TC: Tomografía computerizada.

En la variante conductual se ha descrito la reducción en la conectividad entre diferentes partes del cerebro, que incluyen el lóbulo frontal, el cíngulo anterior, la ínsula, la amígdala, el tálamo medial y el cuerpo estriado ventral; todas estas regiones están implicadas en las emociones (Tabla 1).

El hallazgo característico de la APPvs es la atrofia del polo temporal anterior dominante.

El hallazgo característico de la afasia progresiva primaria variante no fluente agramatical (APPvnf) es atrofia del giro frontal inferior izquierdo, cercano al área perisilviana e involucrando al área de Broca.

En resumen, los estudios funcionales de conectividad nos han demostrado que en la DFT las áreas anatómicas involucradas se ven afectadas con fenotipos específicos ${ }^{18}$.

Reconocer las alteraciones de las conexiones en la sustancia blanca estructural es de particular interés en la comprensión de la propagación de la neurodegeneración. El estudio de DTI nos permite la 
visualización de las conexiones estructurales, al medir la dirección (anisotropía fraccional [FA]) y el nivel de difusividad (radial y axial) del agua en los tractos nerviosos. Una FA elevada nos indica fibras conectadas intactas, ya que la difusión del agua está restringida direccionalmente a lo largo de estos tractos. Por lo tanto, una disminución en FA y un aumento en la difusividad sugieren la degeneración de estas fibras direccionales y la pérdida de conectividad estructural dentro del cerebro' ${ }^{19}$.

En la variante conductual los pacientes presentan reducción extendida en FA y un incremento en las medidas de difusividad en los tractos, con conexiones recíprocas a los lóbulos frontal y temporal. Los tractos clave incluyen el fascículo uncinado, la rodilla del cuerpo calloso y el cíngulo. También se han reportado alteraciones en las partes anteriores de los fascículos longitudinales superior e inferior y del fascículo frontooccipital inferior, regiones conectadas de materia gris ${ }^{20}$.

En los pacientes que sufren la variante conductual, con mutación en MAPT, el cíngulo y el fascículo uncinado están particularmente afectados. Se han encontrado anormalidades funcionales y estructurales en el fascículo uncinado en sujetos presintomáticos que tienen la mutación de este gen.

En portadores de la mutación C9orf72 se han demostrado reducciones en FA en los pedúnculos cerebelares superiores ${ }^{21}$.

En la APPvs predominan las anormalidades frontotemporales del lado izquierdo, incluyendo el fascículo uncinado y el fascículo longitudinal inferior.

En la APPvnf hay una disminución en la FA y un incremento en la difusividad, con predominio de anormalidades frontotemporales izquierdas.

De acuerdo con la distribución de la pérdida de volumen de materia gris, las anomalías de la sustancia blanca en las variantes de la APP variante logopénica (APPvl) demuestran predominio frontoparietal izquierdo, incluidos el fascículo longitudinal superior izquierdo y el fascículo arqueado particularmente.

Las mediciones de DTI también parecen ser prometedoras como marcadores para identificar los cambios patogénicos más tempranos en la DFT. Los tractos que demuestran anormalidades están conectados a regiones de pérdida significativa de materia gris en las tres variantes sindrómicas, pero a menudo la integridad de la sustancia blanca precede a los cambios corticales. Esto se ve respaldado por estudios presintomáticos que detectan cambios en la sustancia blanca en ausencia de anormalidades en la sustancia gris ${ }^{22}$.
Otros estudios que se utilizan en la actualidad y en algunas instituciones que cuentan con el equipo requerido son la FDG-PET y la SPECT, métodos de perfusión ampliamente utilizados para investigar los patrones del metabolismo cerebral de la glucosa o la perfusión cerebral como medidas de la disfunción neuronal. La SPECT permite evaluar zonas cerebrales con perfusión sanguínea y otorga información adicional cuando se conocen áreas con atrofia focal, y que pueden o no tener hipoperfusión. Esto se asocia a la enfermedad que la está ocasionando, ya sea EA o DFT. La preservación de perfusión en el lóbulo parietal en la SPECT nos ayuda a diferenciar el diagnóstico de DFT y EA con una sensibilidad del $80 \%$. Los patrones de hipometabolismo tienden a concordar estrechamente con los patrones de atrofia. El hipometabolismo en las regiones frontotemporales comúnmente se encuentra en pacientes con DFTvc, y afecta particularmente a las regiones orbitofrontal y dorso lateral, a la corteza prefrontal medial y a los polos temporales anteriores. En Ios pacientes con APPvs predomina el hipometabolismo del lóbulo temporal, con afectación asimétrica del hemisferio izquierdo en la corteza entorrinal y perirrinal, polos temporales inferiores y amígdala.

Los pacientes con APPvnf tienen un hipometabolismo más pronunciado, particularmente en las regiones frontales izquierdas inferiores.

Los pacientes con APPvl muestran un hipometabolismo frontotemporoparietal izquierdo distribuido, particularmente en los lóbulos temporales en su porción lateral, lóbulos frontales en su región posterior y lateral, junto con las regiones de los núcleos caudados, el cíngulo posterior y el precúneo.

La FDG-PET y la SPECT se han utilizado ampliamente para evaluar los cambios metabólicos regionales en la DFT, y tienen valor en el diagnóstico diferencial y la detección temprana de anomalías.

La ASL (arterial spin labeling) ayuda a evidenciar el flujo sanguíneo cerebral y si existe alguna alteración. Las imágenes de ASL pueden emplearse para evaluar el flujo sanguíneo cerebral usando $\mathrm{H}_{2} \mathrm{O}$ de sangre arterial marcada magnéticamente como marcador endógeno.

Pacientes con DFTvc muestran hipoperfusión bilateral en las regiones frontal anterior y cingular, mientras que los pacientes con EA presentan un perfil más posterior que incluye las regiones parietales medial, cingulada posterior y el precúneo. Recientemente se ha demostrado que la presencia de hipoperfusión cingulada posterior es un marcador de diagnóstico diferencial y 
Rev Mex Neuroci. 2018;19

Tabla 2. Hallazgos estructurales y funcionales en la imagen de los pacientes con DFT

\begin{tabular}{|c|c|c|}
\hline \multirow[t]{2}{*}{ Variante } & \multicolumn{2}{|l|}{ Hallazgos } \\
\hline & RM & PET/SPECT \\
\hline DFTvc & $\begin{array}{l}\text { Atrofia en la corteza prefrontal, regiones temporales } \\
\text { anteriores, cíngulo anterior, estriado e ínsula }\end{array}$ & $\begin{array}{l}\text { Disminución de FA en fascículo uncinado, genu del cuerpo calloso } \\
\text { y cíngulo, fascículos longitudinales superiores e inferiores, y } \\
\text { fascículo frontooccipital inferior }\end{array}$ \\
\hline DFTvnf & $\begin{array}{l}\text { Pérdida cortical predominante del hemisferio } \\
\text { izquierdo; atrofia que afecta predominantemente a } \\
\text { la parte opercular en la circunvolución frontal } \\
\text { inferior, y se extiende a las cortezas prefrontal y } \\
\text { temporal, caudado y atrofia del putamen } \\
\text { bilateralmente }\end{array}$ & $\begin{array}{l}\text { FA disminuida en fascículo uncinado izquierdo, fascículo folicular } \\
\text { longitudinal inferior, cuerpo calloso y cíngulo; preservación } \\
\text { notable de la sustancia blanca occipital, tallo cerebral y cerebelo }\end{array}$ \\
\hline DFTvs & $\begin{array}{l}\text { Pérdida cortical predominante del hemisferio } \\
\text { izquierdo; pérdida de materia gris } \\
\text { predominantemente en circunvoluciones temporales } \\
\text { y fusiformes inferiores, polo temporal, corteza } \\
\text { parahipocampal, corteza entorrinal; las pérdidas se } \\
\text { extienden a las cortezas cingulada anterior, } \\
\text { orbitofrontal, frontal inferior e insular }\end{array}$ & $\begin{array}{l}\text { FA disminuida en el fascículo longitudinal superior izquierdo, } \\
\text { cuerpo calloso, regiones izquierdas del cíngulo orbitofrontal } \\
\text { izquierdo, frontal inferior, temporal anterior y parietal inferior } \\
\text { de la sustancia blanca }\end{array}$ \\
\hline APPvl & $\begin{array}{l}\text { Atrofia del cíngulo posterior y temporoparietal } \\
\text { izquierdo }\end{array}$ & $\begin{array}{l}\text { Pérdidas notables de la sustancia blanca en regiones } \\
\text { frontoparietales izquierdas, incluyendo fascículo longitudinal } \\
\text { superior izquierdo y fascículo arqueado }\end{array}$ \\
\hline
\end{tabular}

RM: resonancia magnética; DFT: demencia fronto-temporal, DFTvc: demencia fronto-temporal variante conductual, DFTvnf: demencia fronto-temporal variante no fluente, DFTvs: Demencia frontotemporal variante semántica, APPvl: Afasia progresiva primaria variante logopénica.

resulta sensible en las primeras etapas para diferenciar la EA con DFTvc ${ }^{23}$.

Existen otros métodos diagnósticos que nos permiten generar una imagen molecular al momento, como es el caso de la PET amiloide. El desarrollo de marcadores de imágenes basados en PET, que se unen y muestran la distribución de agregaciones anormales de amiloide en el cerebro, ha demostrado ser valioso para visualizar la neuropatología in vivo ${ }^{24}$.

Varios estudios han demostrado la utilidad diagnóstica de las imágenes de amiloide para ayudar a la diferenciación de EA de DFT, con una sensibilidad y una especificidad relativamente altas, dado que las placas de Abeta son un marcador distintivo de EA, y no están dentro del espectro patológico de la DFT. La mayoría de los pacientes con DFT revelan hallazgos de imagen negativos para Abeta, con la notable excepción de los pacientes con afasia progresiva primaria variante logopénica (APPvl), que generalmente tienen enfermedad subyacente de EA y, por lo tanto, retención del marcador amiloide (tabla 2). Varios estudios también revelan un pequeño porcentaje de pacientes con DFTvc, APPvs y APPvnf con PET amiloide positivo ${ }^{25}$.

En resumen, la PET amiloide es de utilidad para diferenciar la EA de la DFT incluso en presencia de síntomas clínicos superpuestos. Además, puede resultar útil para detectar presentaciones de DFT atípicas con
EA subyacente o enfermedad mixta. Su valor es mayor en individuos más jóvenes debido a la alta prevalencia de placas amiloide en poblaciones más viejas.

El desarrollo de estos marcadores moleculares en la PET ha dado paso a que se puedan buscar ligandos selectivos de ciertas enfermedades, como es el caso del ligando de tau en la DFT. El reciente desarrollo de ligandos de PET selectivos para la para el marcaje de tau tiene un claro potencial para mejorar el diagnóstico diferencial en la DFT, así como en la estadificación de la carga de tau dentro de las taupatías. Existen varios desafíos para las imágenes tau: primero, los agregados tau son principalmente intracelulares, por lo que el marcador debe cruzar con éxito la barrera hematoencefálica y la membrana celular para alcanzar su objetivo. Además, las seis isoformas diferentes de tau presentes en combinaciones alteradas en diferentes fenotipos clínicos y agregados de tau están sujetas a una variedad de modificaciones postraduccionales que conducen a diversas conformaciones ultraestructurales. Estos y otros más factores han impedido que esta modalidad sea una opción viable para el diagnóstico ${ }^{26}$.

En conclusión, el campo de la neuroimagen en la DFT ha logrado avances significativos en los últimos años, los cuales han sido impulsados por una mejor comprensión de la genética subyacente y la enfermedad, junto con las mejoras en las imágenes y, en particular, 
Tabla 3. Características distintivas de las principales variedades de DFT. Finalmente, el paciente termina en un mutismo acinético en el transcurso de cuatro a siete años de evolución

\begin{tabular}{|l|l|l|}
\hline Variante & Manifestaciones clínicas & Topografía \\
\hline DFTvc & $\begin{array}{l}\text { Apatía, desinhibición, conductas obsesivas y estereotipadas, pérdida de } \\
\text { empatía y simpatía, aplanamiento afectivo, desaliño, comportamiento social } \\
\text { inadecuado, y dificultad para el pensamiento abstracto, planeación y resolución } \\
\text { de problemas }\end{array}$ & Atrofia frontal bilateral \\
\hline APPvnf & $\begin{array}{l}\text { Habla no fluente con agramatismos, anomia, parafasias fonológicas de inicio } \\
\text { insidioso y progresión gradual; disartria, acalculia, apraxia ideomotora, lectura } \\
\text { con paralexias fonéticas y escritura con errores ortográficos }\end{array}$ & $\begin{array}{l}\text { Atrofia frontotemporal asimétrica } \\
\text { con predominio del hemisferio } \\
\text { izquierdo, principalmente del giro } \\
\text { frontal inferior }\end{array}$ \\
\hline APPvs & $\begin{array}{l}\text { Habla fluente con anomia y deterioro en la comprensión de las palabras, } \\
\text { parafasias semánticas, circunloquios, agnosia de objetos (visual) y } \\
\text { caras (prosopagnosia), dislexia superficial o disgrafía, repetición alterada y } \\
\text { deterioro en la producción de habla espontánea }\end{array}$ & $\begin{array}{l}\text { Atrofia temporal anterior bilateral, } \\
\text { con predominio izquierdo }\end{array}$ \\
\hline
\end{tabular}

el desarrollo de imágenes moleculares de tau con mayor afección en áreas específicas (Tabla 3).

\section{Otras posibilidades diagnósticas}

Recientemente ha surgido la idea de que estas enfermedades podrían estudiarse en la piel, dado que el cerebro y la piel tienen origen ectodérmico. Suzuki, et al. han informado de la expresión de TDP-43 en la epidermis de pacientes con esclerosis lateral amiotrófi$\mathrm{ca}^{27}$. Nuestro grupo de investigación tiene el objetivo de evaluar la presencia de TDP-43 y Tau en la epidermis de pacientes con EA, DFT, ALS y controles sanos utilizando anticuerpos monoclonales en secciones de piel incluidas en parafina. Como se revisó anteriormente, TDP-43 y las proteínas Tau son posibles biomarcadores para la DFT y la EA, y anteriormente demostramos la presencia en la piel de proteínas relacionadas con otras enfermedades neurodegenerativas ${ }^{28}$.

Es probable que mediante inmunohistoquímica con anti-TDP-43 y anti-Tau en biopsias de piel pueda desarrollarse un biomarcador útil expresado en la epidermis útil para el estudio del espectro de estas enfermedades ${ }^{29}$ (Fig. 2).

\section{Tratamiento y manejo}

El tratamiento de este tipo de demencia es sintomático, ya que no existe un medicamento que evite el proceso neurodegenerativo propio de la enfermedad. La educación familiar o del cuidador y el tratamiento no farmacológico de soporte en cuestiones de seguridad y comportamiento son esenciales. Sin embargo, las estrategias no farmacológicas se consideran la intervención preferida antes de recurrir a terapias farmacológicas que pueden exacerbar las comorbilidades

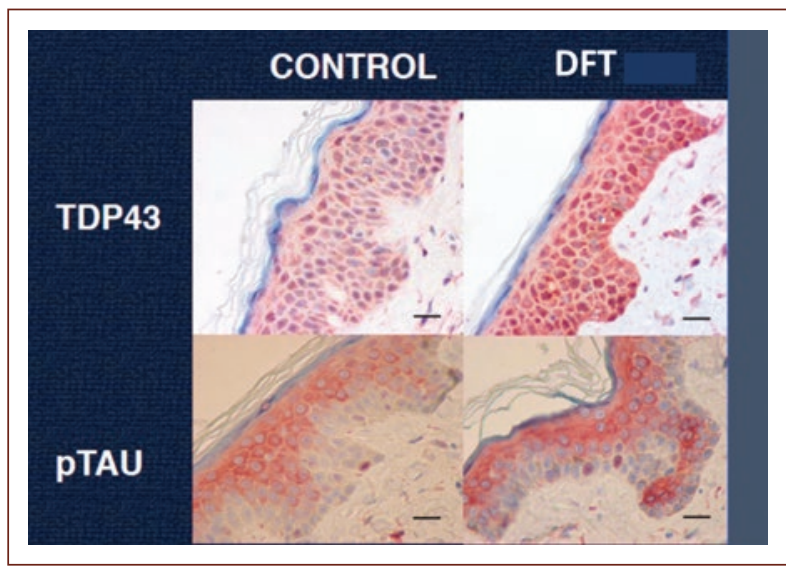

Figura 2. Secciones epidérmicas teñidas para TDP-43 y p-Tau. Observamos un patrón de inmunopositividad similar para TDP-43, con inclusiones en el núcleo y el citoplasma. Sin embargo, para $p$-Tau existe una inmunopositividad citoplasmática difusa. Escala de $10 \mathrm{~mm}$.

médicas que afectan a pacientes más ancianos. El objetivo principal de las intervenciones no farmacológicas es prevenir conductas disruptivas, aliviar los síntomas y disminuir la angustia del cuidador.

\section{Tratamiento no farmacológico}

Los enfoques ambientales apuntan a disminuir la irritabilidad, la agresividad y la ansiedad que surgen de la dificultad de los pacientes para procesar la información de los estímulos diarios. Estas intervenciones incluyen reducir el ruido, limitar la sobreestimulación, simplificar los parámetros sociales, al limitar la interacción a pequeños grupos de personas, y facilitar la eliminación de actividades diarias complicadas que pueden confundir y, por lo tanto, agitar a los pacientes. La introducción 
de juegos o viejos pasatiempos puede reducir la desinhibición y el comportamiento inapropiado. El ejercicio también reduce los síntomas conductuales.

La terapia cognitiva conductual se está estudiando actualmente como una posible opción para modular el comportamiento ${ }^{30}$.

\section{Tratamiento farmacológico}

Normalmente se utilizan los inhibidores selectivos de la recaptura de serotonina para mejorar los trastornos de la conducta, la desinhibición, la agitación o la irritabilidad y la compulsión a ingerir alimentos ricos en carbohidratos. Estos medicamentos no tienen efecto sobre los síntomas cognitivos ${ }^{31}$.

La psicosis y la agresión pueden requerir medicamentos neurolépticos, como la quetiapina, olanzapina o risperidona (aunque las dos últimas se evitan si el paciente tiene parkinsonismo asociado). Se ha sugerido que los antipsicóticos son más útiles para tratar los síntomas psicóticos en pacientes con el subtipo C9orf72 de la DFT, pero una vez más los datos están limitados por un mayor riesgo de efectos secundarios extrapiramidales y morbilidad en pacientes con demencia, lo que impide la utilización segura de estos neurolépticos ${ }^{32}$.

Los estudios con la memantina o los inhibidores colinesterásicos no han mostrado efectos contundentes sobre la cognición, e incluso se han reportado casos en los que se produce un empeoramiento de los síntomas con el uso de donepecilo ${ }^{33}$.

Algunos informes muestran efectos positivos de la clomipramina y carbamacepina en el tratamiento de las conductas compulsivas y de una conducta sexual inapropiada ${ }^{34,35}$.

Compuestos con propiedades aminérgicas, como la selegilina y la dextroanfetamina, compensan la disfunción dopaminérgica involucrada en los síntomas de la DFT, como la apatía.

Algunos estudios demuestran que el topiramato es efectivo como agente antiimpulsivo.

Debido a las limitadas intervenciones psicofarmacológicas contemporáneas disponibles para los médicos, se están investigando varios enfoques novedosos para desarrollar tratamientos más efectivos para los síntomas de la DFT. Uno de estos enfoques es el uso del neuropéptido oxitocina para ayudar en el comportamiento mediador, y mejorar la apatía y la empatía en pacientes con APPvs. Bebidas que contienen omega-3, complejo B, colina y antioxidantes han pudieran mejorar los síntomas. Otro estudio sugirió que la agomelatina, un agonista del receptor de la melatonina, puede mejorar la apatía en pacientes con $\mathrm{DFT}^{36}$.

Los ensayos clínicos con nuevos fármacos dirigidos a genes específicos, proteínas y vías relacionadas con tau, progranulina y C9orf72 prevén un mejor futuro cercano. Por ejemplo, un método que se ha propuesto es elevar los niveles de PGRN a través de la manipulación molecular de las vías biológicas utilizando ácido hidroxámico suberoilanilida, tratamiento con andrógenos y bafilomicina $A 1$.

En resumen, debido a los datos y el poder limitado del tratamiento farmacológico de la DFT, las intervenciones no farmacológicas y la ayuda del cuidador son las opciones de manejo más viables ${ }^{37}$.

\section{Perspectivas}

Hay un largo camino por recorrer en la comprensión de la DFT. Como un problema neurodegenerativo, estas enfermedades se correlacionan con los sitios anatómicos del cerebro afectados con proteinopatías específicas. Las manifestaciones clínicas están relacionadas con los hallazgos de imagen. Es posible que podamos ver los mismos hallazgos que el cerebro muestra con el uso de biomarcadores de la piel, "como un espejo del cerebro", ya que ambos tejidos tienen un origen neuroectodérmico. Podría ser una forma fantástica de estudiar desde la fisiopatología hasta la respuesta terapéutica cuando podamos tener un tratamiento específico para la DFT.

\section{Conclusiones}

La DFT es la demencia neurodegenerativa más frecuente antes de los 65 años. Sus principales manifestaciones clínicas son la afección del lenguaje y las alteraciones del comportamiento. La mayoría de los casos son esporádicos, y hay tres principales mutaciones cuando es genética: MAPT (cromosoma 17q21.32), PGRN (17q21.32) y C9orf72 (9p.21.2). El conocimiento del problema ha permitido hacer un diagnóstico de mayor precisión basado en la clínica: a) aspectos conductuales: apatía, desinhibición, conductas obsesivo-compulsivas, estereotipias, glotoneria, desaliño, y b) aspectos del lenguaje: pérdida de la fluencia verbal, agramatismo, parafasias fonológicas, anomia, apraxia en el discurso, diversas agnosias, dislexia, agrafia, parafasias semánticas y frecuentemente encontramos signos de liberación frontal que progresará al final hasta el mutismo. La imagen puede mostrar la afección estructural principalmente de atrofia frontotemporal, que puede ser francamente asimétrica 
si la afección es más de lenguaje (izquierda); la atrofia es más temporal si la APP es más semántica, y frontal bilateral si la variante de presentación es conductual. No hay un tratamiento específico, por lo que debemos conformarnos con dar manejo sintomático para mejorar la calidad de vida del paciente (antidepresivos, antipsicóticos atípicos). El uso de memantina y anticolinesterásicos es controversial. El pronóstico es malo a mediano plazo. La única forma de realizar un diagnóstico definitivo es teniendo el tejido cerebral y demostrando la presencia de cuerpos de Pick con proteína p-Tau agregada, microgliosis, astrogliosis, y acumulación de FUS y TDP-43. Nuestro grupo de investigación ha propuesto la piel como un posible sitio de estudio de las proteinopatías en sujetos vivos para demostrar la presencia de la proteína $p$-Tau, TDP-43 y FUS en sujetos afectados, lo que nos permitiría conocer no sólo su presencia, sino también conocer mejor la fisiopatogenia, estudiar posibles tratamientos e incluso evaluar su respuesta.

\section{Bibliografía}

1. Bang J, Spina S, Miller BL. Frontotemporal dementia. Lancet. 2015; 386(10004):1672-82.

2. Duthey B. Background paper 6.11: Alzheimer disease and other dementias. A Public Health Approach to Innovation. 2013:1-74.

3. Onyike CU, Diehl-Schmid J. The epidemiology of frontotemporal dementia. Int Rev Psychiatry. 2013;25(2):130-7.

4. Chare L, Hodges JR, Leyton CE, McGinley C, Tan RH, Kril JJ, et al. New criteria for frontotemporal dementia syndromes: clinical and pathological diagnostic implications. J Neurol Neurosurg Psychiatry. 2014;85(8):865-70.

5. Rascovsky K, Hodges JR, Kipps CM, Johnson JK, Seeley WW, Méndez MF, et al. Diagnostic criteria for the behavioral variant of frontotemporal dementia (bvDFT): current limitations and future directions. Alzheimer Dis Assoc Disord. 2007;21(4):S14-8.

6. Harris JM, Gall C, Thompson JC, Richardson AM, Neary D, du Plessis D, et al. Classification and pathology of primary progressive aphasia. Neurology. 2013;81(21):1832-9.

7. Bonner MF, Sharon A, Grossman M. The new classification of primary progressive aphasia into semantic, logopenic, or nonfluent/agrammatic variants. Curr Neurol Neurosci Rep. 2010;10(6):484-90.

8. Gorno-Tempini ML, Hillis AE, Weintraub S, Kertesz A, Méndez M, Cappa SF, et al. Classification of primary progressive aphasia and its variants. Neurology. 2011;76(11):1006-14.

9. Riedl L, Mackenzie IR, Förstl H, Kurz A, Diehl-Schmid J. Frontotemporal lobar degeneration: current perspectives. Neuropsychiatr Dis Treat. 2014;10:297-310.

10. Seelaar H, Rohrer JD, Pijnenburg YA, Fox NC, van Swieten JC. Clinical, genetic and pathological heterogeneity of frontotemporal dementia: a review. J Neurol Neurosurg Psychiatry. 2010;82(5):476-86.

11. Majounie E, Renton AE, Mok K, Dopper EG, Waite A, Rollinson S, et al. Frequency of the C9orf72 hexanucleotide repeat expansion in patients with amyotrophic lateral sclerosis and frontotemporal dementia: a cross-sectional study. Lancet Neurol. 2012;11(4):323-30.

12. Coppola G, Chinnathambi S, Lee JJ, Dombroski BA, Baker MC, Soto-Ortolaza Al, et al. Evidence for a role of the rare p. A152T variant in MAPT in increasing the risk for DFT-spectrum and Alzheimer's diseases. Hum Mol Genet. 2012;21(15):3500-12.

13. Mackenzie IR. The neuropathology and clinical phenotype of FTD with progranulin mutations. Acta neuropathol. 2007;114(1):49-54.

14. DeJesús-Hernández M, Mackenzie IR, Boeve BF, Boxer AL, Baker M, Ruthenford NJ, et al. Expanded GGGGCC hexanucleotide repeat in non- coding region of C9ORF72 causes chromosome 9p-linked FTD and ALS Neuron. 2011;72(2):245-56.

15. Schröder R, Watts GD, Mehta SG, Evert BO, Broich P, Fliessbach K, et al. Mutant valosin-containing protein causes a novel type of frontotemporal dementia. Ann Neurol. 2005;57(3):457-61.

16. Snowden JS, Adams J, Harris J, Thompson JC, Rollinson S, Richardson A, et al. Distinct clinical and pathological phenotypes in frontotemporal dementia associated with MAPT, PGRN and C9orf72 mutations. Amyotroph Lateral Scler Frontotemporal Degener. 2015;16(7-8):497-505.

17. Whitwell JL, Weigand SD, Boeve BF, Senjem ML, Gunter JL, DeJesús-Hernández $\mathrm{M}$, et al. Neuroimaging signatures of frontotemporal dementia genetics: C9ORF72, tau, progranulin and sporadics. Brain 2012; 135(3):794-806.

18. Whitwell JL, Weigand SD, Boeve BF, Senjem ML, Gunter JL, DeJesús-Hernández $\mathrm{M}$, et al. Distinct profiles of brain atrophy in frontotemporal lobar degeneration caused by progranulin and tau mutations. Neuroimage 2010;53(3):1070-6.

19. James JS, Kumari SR, Sreedharan RM, Thomas B, Radhkrishnan A, Kesavadas C. Analyzing functional, structural, and anatomical correlation of hemispheric language lateralization in healthy subjects using functional MRI, diffusion tensor imaging, and voxel-based morphometry. Neurol India. 2015;63(1):49-57.

20. Whitwell JL, Avula R, Senjem ML, Kantarci K, Weigans SD, Samikoglu A, et al. Gray and white matter water diffusion in the syndromic variants of frontotemporal dementia. Neurology. 2010;74(16):1279-87.

21. Mahoney C, Zhang H, Malone I, Nicholas J, Schmitz N, Schott J, et al. Tracking white matter degeneration in frontotemporal dementia using serial diffusion tensor imaging. Alzheimer's \& Dementia: The Journal of the Alzheimer's Association. 2013;9(4):56-7.

22. Lillo P, Mioshi E, Burrell JR, Kiernan MC, Hodges JR, Hornberger M. Grey and white matter changes across the amyotrophic lateral sclerosis-frontotemporal dementia continuum. PloS One. 2012;7(8):e43993.

23. Foster NL, Heidebrink JL, Clark CM, Jagust WJ, Arnold SE, Barbas NR, et al. FDG-PET improves accuracy in distinguishing frontotemporal dementia and Alzheimer's disease. Brain. 2007;130(10):2616-35.

24. Diehl J, Grimmer T, Drzezga A, Riemenschneider M, Förstl H, Kurz A. Cerebral metabolic patterns at early stages of frontotemporal dementia and semantic dementia. A PET study. Neurobiol Aging. 2004;25(8):1051-6.

25. Engler H, Santillo AF, Wang SX, Lindau M, Savitcheva I, Nordberg A, et al. In vivo amyloid imaging with PET in frontotemporal dementia. Eur J Nucl Med Mol Imaging. 2008;35(1):100-6.

26. Rabinovici GD Furst AJ, O'Neil JP, Racine CA, Mormino EC, Baker SL, et al. 11C-PIB PET imaging in Alzheimer disease and frontotemporal lobar degeneration. Neurology 2007;68(15):1205-12.

27. Suzuki M, Mikami H, Watanabe T, Yamano T, Yamazaki T, Nomura M, et al. Increased expression of TDP-43 in the skin of amyotrophic lateral sclerosis. Acta Neurol Scand. 2010;122(5):367-72.

28. Rodríguez-Leyva I, Chi-Ahumada EG, Carrizales J, Rodríguez-Violante M, Velázquez-Osuna S, Medina-Mier V, et al. Parkinson disease and progressive supranuclear palsy: protein expression in skin. Ann Clin Transl Neurol. 2016;3(3):191-9.

29. Rodríguez-Leyva I, Calderón-Garcidueñas AL, Jiménez-Capdeville ME. Plegamiento anormal de proteínas y neurodegeneración. Revista Mexicana de Neurociencia. 2015;16(1):51-72.

30. Perry RJ, Miller BL. Behavior and treatment in frontotemporal dementia. Neurology. 2001;56(Suppl 4):S46-51.

31. Moretti R, Torre P, Antonello RM, Cazzato G, Bava A. Frontotemporal dementia: paroxetine as a possible treatment of behavior symptoms. Eur Neurol. 2003;49(1):13-9.

32. Shinagawa S, Nakajima S, Plitman E, Graff-Guerrero A, Mimura M, Nakayama K, et al. Psychosis in frontotemporal dementia. J Alzheimers Dis. 2014;42(2):485-99.

33. Méndez MF, Shapira JS, McMurtray A, Licht E. Preliminary findings: behavioral worsening on donepezil in patients with frontotemporal dementia. Am J Geriatr Psychiatry. 2007;15(1):84-7.

34. Jenssen S, Schere D. Treatment and management of epilepsy in the elderly demented patient. Am J Alzheimers Dis Other Demen. 2010;25(1):18-26.

35. Tsai RM, Richard M, Boxer AL. Treatment of frontotemporal dementia. Curr Treat Options Neurol. 2015;16(11):319.

36. Callegari I, Mattei C, Benassi F, Krueger F, Grafman J, Yaldizli Ö, et al. Agomelatine improves apathy in frontotemporal dementia. Neurodegener Dis. 2016;16(5-6):352-6.

37. Li YQ, Tan MS, Yu JT, Tan L. Frontotemporal lobar degeneration: mechanisms and therapeutic strategies. Mol Neurobiol. 2016;53(9):6091-105. 